\title{
A New Approach for Higher Order Difference Equations and Eigenvalue problems via Physical Potentials
}

\author{
Erdal $\mathrm{BAS}^{a}$ and Ramazan OZARSLAN ${ }^{a *}$ \\ ${ }^{a}$ Firat University, Science Faculty, Department of Mathematics, 23119 Elazig/Turkey \\ e-mail: erdalmat@yahoo.com, *ozarslanramazan@gmail.com
}

\begin{abstract}
In this study, we give the variation of parameters method from a different viewpoint for the $N$ th order inhomogeneous linear ordinary difference equations with constant coefficient by means of delta exponential function $e_{p}(t, s)$. Advantage of this new approachment is to enable us to investigate the solution of difference equations in the closed form. Also, the method is supported with three difference eigenvalue problems; the second-order Sturm-Liouville problem, which is called also one dimensional Schrödinger equation, having Coulomb potential, hydrogen atom equation, and the fourth-order relaxation difference equations. We find sum representation of solution for the second order discrete Sturm-Liouville problem having Coulomb potential, hydrogen atom equation, and analytical solution of the fourth order discrete relaxation problem by the variation of parameters method via delta exponential and delta trigonometric functions .
\end{abstract}

Keywords: Schrödinger equation, energy level, difference equation, variation of parameters method, Coulomb potential, hydrogen atom, relaxation equation.

AMS Subject Classification: 39A06; 39A12; 39A70; 35A25.

\section{Introduction}

Variation of parameters method, that is a general method in the solution methods of inhomogeneous linear ordinary differential equations, firstly was introduced by Euler and Lagrange while they were studying the celestial bodies and orbital elements [15, 16.

Later, this method was adapted to solve inhomogeneous linear ordinary difference equations for the following equation types in [12, 13]

$$
x_{n+N}+p_{N-1} x_{n+N-1}+\cdots+p_{0} x_{n}=q(n) .
$$

Recently, delta exponential function has been described in 11 similarly to exponential function in the continuous case. $e^{p t}, p \in \mathbb{R}$, is the solution of the following problem

$$
\begin{aligned}
y^{\prime}(t) & =p y(t), \\
y(0) & =1,
\end{aligned}
$$

and delta exponential function $e_{p}(n, s)$ is the solution of the following problem

$$
\begin{aligned}
\Delta x(n) & =p(n) x(n), \\
x(s) & =1 .
\end{aligned}
$$

Also, delta exponential function $e_{p}(n, s)$ is used to find the homogeneous solution of linear difference equations with constant coefficient as with differential equations and besides, variation of constants formula is given for the first order linear difference equations in 11.

In this study, we generalize the method for $N$ th order inhomogeneous linear ordinary difference equations with constant coefficient by means of delta exponential function $e_{p}(t, s)$ and consider the following equation in a closed form differently from the equation (1)

$$
\Delta^{N} x(n)+r_{N-1} \Delta^{N-1} x(n)+\cdots+r_{0} x_{n}=q(n) .
$$


We present a different approach to the variation of parameters method by means of delta exponential function for higher order inhomogeneous linear ordinary difference equations with constant coefficient. This approachment enables us to investigate the solution of difference equations in the closed form as given in (2), otherwise equation (2) has to be expanded by using binomial expansion of the difference operator

$$
\Delta^{N} x(t)=\sum_{k=0}^{N}(-1)^{k}\left(\begin{array}{c}
N \\
k
\end{array}\right) x(t+N-k) .
$$

In the last section, we give three examples for explaining the method, also specifically we find the representation of solution of discrete Sturm-Liouville problem, which is called also one dimensional Schrödinger equation, having Coulomb potential, discrete hydrogen atom equation, and the fourth-order discrete relaxation equation.

Sturm-Liouville equations play an important role in mathematical physics. Lately, Sturm-Liouville differential and difference equations have been considered similarly to the continuous counterpart $[1-10,14]$.

Hydrogen atom equation is studied by [19, 20, 17, 18. Hydrogen atom equation is used in quantum mechanics for determining energy levels of hydrogen atom [19. Hydrogen atom equation is defined as follows

$$
y^{\prime \prime}+\left(\lambda-\frac{l(l+1)}{x^{2}}+\frac{2}{x}-q(x)\right) y=0 .
$$

Then, let's introduce discrete hydrogen atom equation

$$
\Delta^{2} x(n-1)+\left(\lambda-q(n)+\frac{2}{n}-\frac{l(l+1)}{n^{2}}\right) x(n)=0, n=1, \ldots, b,
$$

where $l$ is a positive integer or zero, $v(n) \in l^{2}[0, b], q(n), b, \lambda$ and $n$ is as defined above, $-q(n)+\frac{2}{n}-$ $\frac{l(l+1)}{n^{2}}$ are called potential function.

Now, let's define briefly Sturm-Liouville operator having Coulomb potential. Motion of electrons moving under the Coulomb potential has importance in quantum theory. This problem is used for finding energy levels for hydrogen atom and single valence electron atoms. Time-dependent Schrödinger equation is as follows

$$
i \tilde{h} \frac{\partial \omega}{\partial t}=-\frac{\tilde{h}^{2}}{2 m} \frac{\partial^{2} \omega}{\partial x^{2}}+U(x, y, z) \omega, \quad \int_{R^{3}}|\omega|^{2} d x d y d z=1,
$$

from here, in consequence of some transformations, we obtain Sturm-Liouville equation having Coulomb potential

$$
-y^{\prime \prime}+\left[\frac{A}{x}+q(x)\right] y=\lambda y,
$$

where $\lambda$ is a parameter which corresponds to the energy [21]. The following problem

$$
\begin{aligned}
-\Delta^{2} x(n-1)+ & \left(\frac{A}{n}+q(n)\right) x(n)=\lambda u(n), n=a, \ldots, b, \\
x(a-1)+h x(a) & =0,
\end{aligned}
$$

is called discrete Sturm-Liouville problem having Coulomb potential. 


\section{Preliminaries}

Definition 1. 11] Let's define regressive functions,

$$
\Re=\left\{p: \mathbb{N}_{a} \rightarrow \mathbb{R} \text { such that } 1+p(n) \neq 0 \text { for } n \in \mathbb{N}_{a}\right\} .
$$

Theorem 2. 11] Let's define delta exponential function. Suppose that $p \in \Re$ and $s \in \mathbb{N}_{a}$, then

$$
e_{p}(n, s)= \begin{cases}\prod_{\tau=s}^{n-1}[1+p(\tau)], & n \in \mathbb{N}_{s} \\ s-1 & n \in \mathbb{N}_{a}^{s-1},\end{cases}
$$

where $\prod_{\tau=a}^{b} \cdot=1$, if $a>b$.

Theorem 3. 11] Suppose that $p, q \in \Re$ and $n, s \in \mathbb{N}_{a}$. Then

(i) $e_{0}(n, s)=1$

(ii) $\Delta e_{p}(n, s)=p(n) e_{p}(n, s)$;

(iii) $e_{p}(n, s) e_{q}(n, s)=e_{p \oplus q}(n, s)$,

where $p \oplus q=p+q+p q$.

Definition 4. [1] Let's define delta sine and cosine functions as follows,

$$
\cos _{p}(n, a)=\frac{e_{i p}(n, a)+e_{-i p}(n, a)}{2}, \sin _{p}(n, a)=\frac{e_{i p}(n, a)-e_{-i p}(n, a)}{2 i},
$$

where $n \in \mathbb{N}_{a}, \pm i p \in \Re$.

Definition 5. [11] Let's define delta integral. Suppose $f: \mathbb{N}_{a} \rightarrow \mathbb{R}$ and $c \leq d, c, d \in \mathbb{N}_{a}$, then

$$
\int_{c}^{d+1} f(n) \Delta n=\sum_{n=c}^{d} f(n)
$$

where $\sum_{n=c}^{d} \cdot=0$, if $c>d$.

Definition 6. 11] If $F(n)$ is delta integral of $f(n)$, then

$$
\int_{a}^{b} f(n) \Delta n=F(b)-F(a) .
$$

Let's consider the second order linear homogeneous ordinary difference equation with constant coefficients as follows,

$$
\Delta^{2} y(n)+p \Delta y(n)+q y(n)=0, n \in \mathbb{N}_{a},
$$

where $p, q \in \mathbb{R}$ hold $p \neq 1+q$.

Theorem 7. 11] Characteristic equation of (3), by the help of delta exponential function, is given by

$$
m^{2}+p m+q=0
$$


let $m_{1}, m_{2}$ are distinct characteristic roots of the characterstic equation, so

$$
y(n)=c_{1} e_{m_{1}}(n, a)+c_{2} e_{m_{2}}(n, a),
$$

where $c_{1}, c_{2}$ are constants, is a general solution of (3) .

Theorem 8. 11] Let the characteristic roots are complex pair, $m_{1,2}=\alpha \pm i \beta, \alpha \neq-1, \beta>0$, so

$$
y(n)=c_{1} e_{\alpha}(n, a) \cos \gamma(n, a)+c_{2} e_{\alpha}(n, a) \sin _{\gamma}(n, a),
$$

where $\gamma=\frac{\beta}{1+\alpha}$, is a general solution of (3).

Theorem 9. 11] Let the characteristic roots are double roots, $m_{1}=m_{2}=r$, so

$$
y(n)=c_{1} e_{r}(n, a)+c_{2}(n-a) e_{r}(n, a),
$$

is a general solution of (3).

\section{Main Results}

\subsection{Analysis of the Method}

Let's reconsider the equation (2);

$$
\Delta^{N} x(n)+r_{N-1} \Delta^{N-1} x(n)+\cdots+r_{0} x_{n}=q(n) .
$$

If we change the variable $x(n)=e_{m}(n, 0)$ and consider the homogeneous part of (2), then we have the following characteristic equation

$$
m^{N}+r_{N-1} m^{N-1}+\cdots+r_{0}=0,
$$

and let its roots are $m_{1}, m_{2}, \ldots, m_{N}$. Hence, we have the homogeneous solution as follows,

$$
x(n)=c_{1} x_{1}(n)+c_{2} x_{2}(n)+\cdots+c_{N} x_{N}(n),
$$

where $x_{1}(n)=e_{m_{1}}(n, 0), x_{2}(n)=e_{m_{2}}(n, 0), \ldots, x_{N}(n)=e_{m_{N}}(n, 0)$ is a linearly independent set of solutions.

From here, let's take a set of new solution functions for the variation of parameters method, $v_{1}(n), v_{2}(n), \ldots, v_{N}(n)$ and so, let's assume that following equation

$$
X(n)=v_{1}(n) x_{1}(n)+v_{2}(n) x_{2}(n)+\cdots+v_{N}(n) x_{N}(n)
$$

is a solution of nonhomogeneous part of (2) .

For finding the parameters $v_{1}(n), v_{2}(n), \ldots, v_{N}(n)$, firstly let's take

$$
\begin{aligned}
\Delta X(n)= & {\left[\Delta v_{1}(n) x_{1}(n+1)+\Delta v_{2}(n) x_{2}(n+1)+\cdots+\Delta v_{N}(n) x_{N}(n+1)\right]+} \\
& {\left[v_{1}(n) \Delta x_{1}(n)+v_{2}(n) \Delta x_{2}(n)+\cdots+v_{N}(n) \Delta x_{N}(n)\right], }
\end{aligned}
$$

let's assume that the first bracketed part at the right hand side of the equation above equals to zero,

$$
\Delta v_{1}(n) x_{1}(n+1)+\Delta v_{2}(n) x_{2}(n+1)+\cdots+\Delta v_{N}(n) x_{N}(n+1)=0,
$$

and so we have,

$$
\Delta X(n)=v_{1}(n) \Delta x_{1}(n)+v_{2}(n) \Delta x_{2}(n)+\cdots+v_{N}(n) \Delta x_{N}(n),
$$


then, let's take the difference of the equality above

$$
\begin{aligned}
\Delta^{2} X(n)= & {\left[\Delta v_{1}(n) \Delta x_{1}(n+1)+\Delta v_{2}(n) \Delta x_{2}(n+1)+\cdots+\Delta v_{N}(n) \Delta x_{N}(n+1)\right]+} \\
& {\left[v_{1}(n) \Delta^{2} x_{1}(n)+v_{2}(n) \Delta^{2} x_{2}(n)+\cdots+v_{N}(n) \Delta^{2} x_{N}(n)\right], }
\end{aligned}
$$

let's assume that

$$
\Delta v_{1}(n) \Delta x_{1}(n+1)+\Delta v_{2}(n) \Delta x_{2}(n+1)+\cdots+\Delta v_{N}(n) \Delta x_{N}(n+1)=0,
$$

so we have

$$
\Delta^{2} X(n)=v_{1}(n) \Delta^{2} x_{1}(n)+v_{2}(n) \Delta^{2} x_{2}(n)+\cdots+v_{N}(n) \Delta^{2} x_{N}(n),
$$

proceeding in this fashion, we have

$$
\Delta^{N} X(n)=v_{1}(n) \Delta^{N} x_{1}(n)+v_{2}(n) \Delta^{N} x_{2}(n)+\cdots+v_{N}(n) \Delta^{N} x_{N}(n) .
$$

If we formulate our assumptions above, we have

$$
\begin{gathered}
\Delta^{k} X(n)=v_{1}(n) \Delta^{k} x_{1}(n)+v_{2}(n) \Delta^{k} x_{2}(n)+\cdots+v_{N}(n) \Delta^{k} x_{N}(n), k=1,2, \ldots, N-1, \\
\Delta^{k} v_{1}(n) \Delta x_{1}(n+1)+\Delta^{k} v_{2}(n) \Delta x_{2}(n+1)+\cdots+\Delta^{k} v_{N}(n) \Delta x_{N}(n+1)=0, k=0,1, \ldots, N-2,
\end{gathered}
$$

if obtained equalities above is written in (2), then we have the following equation system

$$
\begin{aligned}
\Delta v_{1}(n) x_{1}(n+1)+\Delta v_{2}(n) x_{2}(n+1)+\cdots+\Delta v_{N}(n) x_{N}(n+1) & =0, \\
\Delta v_{1}(n) \Delta x_{1}(n+1)+\Delta v_{2}(n) \Delta x_{2}(n+1)+\cdots+\Delta v_{N}(n) \Delta x_{N}(n+1) & =0, \\
& \vdots \\
\Delta v_{1}(n) \Delta^{N-2} x_{1}(n+1)+\Delta v_{2}(n) \Delta^{N-2} x_{2}(n+1)+\cdots+\Delta v_{N}(n) \Delta^{N-2} x_{N}(n+1) & =0, \\
\Delta v_{1}(n) \Delta^{N-1} x_{1}(n+1)+\Delta v_{2}(n) \Delta^{N-1} x_{2}(n+1)+\cdots+\Delta v_{N}(n) \Delta^{N-1} x_{N}(n+1) & =q(n) .
\end{aligned}
$$

If we solve this system by Cramer rule, we have the Casoratian,

$$
\operatorname{det}\left|\begin{array}{cccc}
x_{1}(n+1) & x_{2}(n+1) & \cdots & x_{N}(n+1) \\
\Delta x_{1}(n+1) & \Delta x_{2}(n+1) & \cdots & \Delta x_{N}(n+1) \\
\vdots & \vdots & \ddots & \vdots \\
\Delta^{N-1} x_{1}(n+1) & \Delta^{N-1} x_{2}(n+1) & \cdots & \Delta^{N-1} x_{N}(n+1)
\end{array}\right|=W\left(x_{1}, x_{2}, \cdots, x_{N}\right)(n+1)
$$

and assuming the Casoratian is different from zero. Let $W_{i}$ correspond the determinant of the $i$ th column of the Casoratian with the column $(0,0,0, \ldots, 0,1)$ and so, solution of the system as follows

$$
\Delta v_{1}(n)=\frac{q(n) W_{1}(n)}{W(n+1)}, \Delta v_{2}(n)=\frac{q(n) W_{2}(n)}{W(n+1)}, \cdots, \Delta v_{N}(n)=\frac{q(n) W_{N}(n)}{W(n+1)},
$$

from here we have the parameters as follows

$$
v_{1}(n)=\int \frac{q(n) W_{1}(n)}{W(n+1)} \Delta n, v_{2}(n)=\int \frac{q(n) W_{2}(n)}{W(n+1)} \Delta n, \cdots, v_{N}(n)=\int \frac{q(n) W_{N}(n)}{W(n+1)} \Delta n .
$$

Finally, the particular solution is as follows

$$
X(n)=x_{1}(n) \int \frac{q(n) W_{1}(n)}{W(n+1)} \Delta n+x_{2}(n) \int \frac{q(n) W_{2}(n)}{W(n+1)} \Delta n+\cdots+x_{N}(n) \int \frac{q(n) W_{N}(n)}{W(n+1)} \Delta n .
$$




\subsection{Numerical Results and Discussions of Some Discrete Eigenvalue Problems Having Physical Potential}

In this section, a new version of the variation of parameters method is applied by using delta exponential function. First of all, we consider the second-order Sturm-Liouville problem, which is called also one dimensional Schrödinger equation, having Coulomb potential, hydrogen atom equation, and the fourth-order relaxation difference equations. We find sum representation of solution for the second order discrete Sturm-Liouville problem having Coulomb potential, hydrogen atom equation, and analytical solution of the fourth order discrete relaxation problem by means of variation of parameters method by using delta exponential function.

\subsubsection{Discrete Hydrogen Atom Equation and Discrete Sturm-Liouville Equation Having Coulomb Potential}

First of all, let's consider the following second order hydrogen atom equation,

$$
-\Delta^{2} x(n-1)+\left(\frac{A}{n}+q(n)\right) x(n)=\lambda x(n),
$$

with the boundary conditions,

$$
x(0)=x(b)=0,
$$

has a unique solution $x(n)$ as follows, $n \in[0, b], n$ is a finite integer, $x(n) \in l^{2}[0 . b]$

$$
x(n, \lambda)=\sin n \theta+\frac{1}{\sin \theta} \int_{1}^{n+1}\left(\frac{A}{s}+q(s)\right) x(s) \sin (n-s) \theta \Delta s .
$$

Secondly, let's consider the following second order Sturm-Liouville difference equation having Coulomb potential,

$$
-\Delta^{2} x(n-1)+\left(-q(n)+\frac{2}{n}-\frac{l(l+1)}{n^{2}}\right) x(n)=\lambda x(n),
$$

with the boundary conditions (8) , has a unique solution $x(n)$ as follows, $n \in[0, b], n$ is a finite integer, $x(n) \in l^{2}[0 . b]$

$$
x(n, \lambda)=\sin n \theta+\frac{1}{\sin \theta} \int_{1}^{n+1}\left(-q(s)+\frac{2}{s}-\frac{l(l+1)}{s^{2}}\right) x(s) \sin (n-s) \theta \Delta s .
$$

Fig1: Eigenfunctions for the problem (7)-(8)

Fig2: Eigenfunctions for the problem (9)-(8) 
Fig3:Comparison of the eigenfunctions for the problems (7)-(8) and (9)-(8)

\begin{tabular}{|c|c|c|c|c|c|c|}
$n$ & $x(n)$ for Hydrogen & $x(n)$ for Coulomb & $n$ & $x(n)$ for Hydrogen & $x(n)$ for Coulomb \\
1 & 0.866025 & 0.866025 & 1 & 0.707107 & 0.707107 \\
2 & 2.59808 & 5.19615 & 2 & 2.41421 & 4.53553 \\
3 & 4.86821 & 10.6024 & 3 & 5.62132 & 11.182 \\
4 & 6.70353 & 11.5276 & 4 & 10.6548 & 17.7341 \\
5 & 6.86296 & 5.24802 & 5 & 17.4379 & 20.5481 \\
6 & 4.60124 & -4.77228 & 6 & 25.2922 & 17.227 \\
13 & 6.18061 & -7.29527 & 13 & -7.27102 & 6.52242 \\
14 & 4.53803 & -11.2966 & 14 & -22.8474 & 16.3393 \\
15 & -0.105595 & -5.75247 & 15 & -32.7782 & 19.1177 \\
16 & -4.67793 & 4.67243 & 16 & -34.1566 & -17.9177 \\
21 & 5.26773 & -9.75089 & 21 & 32.7366 & 2.89076 \\
23 & -3.99289 & 8.78504 & 23 & 19.0974 & 19.0276 \\
24 & -6.01193 & 10.8038 & 24 & 0.835118 & 15.2384 \\
25 & -3.49672 & 3.43627 & 25 & -17.7111 &
\end{tabular}

Fig4: Comparison of datas in Table1

Fig5: Comparison of datas in Table2 Suppose that $q(n)=\frac{1}{\sqrt{n}}, A=1, l=2$ in the figures and tables above. 
Proof. Firstly, we study to find the general solution of the equation (77) by the variation of parameters method without using the boundary conditions. Homogenous part of (7) is as follows,

$$
\Delta^{2} x(n-1)+\lambda x(n)=0 .
$$

By using delta exponential function, we have the characteristic equation,

$$
\frac{m^{2}}{1+m}+\lambda=0
$$

and characteristic roots are as follows,

$$
m_{1,2}=\frac{-\lambda \pm \sqrt{\lambda(\lambda-4)}}{2},
$$

where $m_{1,2} \in \Re$. So, the homogeneous solution is found from (4) as follows,

$$
x_{h}(n)=c_{1} e_{m_{1}}(n, 0)+c_{2} e_{m_{2}}(n, 0) .
$$

Then, if we apply the variation of parameters method, lineary independent solutions is found as $e_{m_{1}}(n, 0)$, $e_{m_{2}}(n, 0)$.

From here, if we take the constants as parameters $v_{1}(n)$ and $v_{2}(n)$, then we find the variables by Cramer rule, let $q_{1}(n)=\frac{A}{n}+q(n)$

$$
\Delta v_{1}(n-1)=\frac{\left|\begin{array}{cc}
-q_{1}(n) x(n) & \Delta e_{m_{2}}(n, 0) \\
0 & e_{m_{2}}(n, 0)
\end{array}\right|}{W\left(e_{m_{1}}(n, 0), e_{m_{2}}(n, 0)\right)}
$$

where $W$ is Casoratian,

$$
\begin{aligned}
W\left(e_{m_{1}}(n, 0), e_{m_{2}}(n, 0)\right) & =e_{m_{1} \oplus m_{2}}(n, 0)\left(m_{2}-m_{1}\right), \\
& =-\sqrt{\lambda(\lambda-4)} .
\end{aligned}
$$

Hence,

$$
v_{1}(n)=\frac{1}{\sqrt{\lambda(\lambda-4)}} \int_{0}^{n+1} q_{1}(s) x(s) e_{m_{2}}(s, 0) \Delta s
$$

Similarly,

$$
v_{2}(n)=-\frac{1}{\sqrt{\lambda(\lambda-4)}} \int_{0}^{n+1} q_{1}(s) x(s) e_{m_{1}}(s, 0) \Delta s .
$$

Finally, the general solution is found by

$x(n)=c_{1} e_{m_{1}}(n, 0)+c_{2} e_{m_{2}}(n, 0)+\frac{1}{\sqrt{\lambda(\lambda-4)}} \int_{0}^{n+1} q_{1}(s) x(s)\left[e_{m_{1}}(s, 0) e_{m_{2}}(n, 0)-e_{m_{2}}(s, 0) e_{m_{1}}(n, 0)\right] \Delta s$.

Now, let's continue to the proof by using the boundary conditions (8) . Homogeneous solution is as in the equality (11). For finding untrivial solution, we analyze the eigenvalue $\lambda$ in four cases, these are

i) $\lambda=0$

ii) $\lambda=4$,

iii) $\lambda>0$ and $\lambda<4$,

iv) $0<\lambda<4$. 
We have trivial solutions for the first two cases, we arrive at a contradiction for the third case and finally, if $0<\lambda<4$, then we have untrivial solution. The characteristic roots are complex pair and taking $\lambda=2-2 \cos \theta$, then we have

$$
m_{1,2}=(-1+\cos \theta) \pm i \sin \theta
$$

So, homogeneous solution is as follows by (5)

$$
x(n)=c_{1} e_{\alpha}(n, 0) \cos _{\gamma}(n, 0)+c_{2} e_{\alpha}(n, 0) \sin _{\gamma}(n, 0),
$$

where $\alpha=-1+\cos \theta, \beta=\sin \theta, \gamma=\tan \theta$. If we insert $\alpha, \beta, \gamma$ in the equality above and use Theorem 2 and Definition 4, then we have the homogenous solution of (7)

$$
x(n)=c_{1} \cos n \theta+c_{2} \sin n \theta .
$$

Then, it is easily found the general solution (10) by the variation of parameters method.

Similarly, representation of solution is obtained for (9)

\subsubsection{Fourth Order Relaxation Difference Equation}

Let's consider the following the fourth order relaxation difference equation,

$$
\Delta^{4} x(n)-\lambda x(n)=q(n),
$$

with the initial conditions

$$
x(0)=x(1)=x(2)=x(3)=1 .
$$

The problem (12) - (13) has a unique solution as follows, $n \in[0, b], n$ is a finite integer, $x(n) \in l^{2}[0 . b]$

$$
\begin{aligned}
& x(n)=\frac{\left((1-i s)^{n}+(1+i s)^{n}\right)\left(s^{6}+q_{0}-s^{2}\left(1+q_{0}\right)-2 q_{1}+q_{2}\right)}{4 s^{2}\left(-1+s^{4}\right)}+ \\
& \frac{1}{4 s^{3}\left(-1+s^{4}\right)}(1+s)^{n}\left(s^{7}+q_{0}-s^{3}\left(1+q_{0}\right)+s^{2}\left(q_{0}-q_{1}\right)-3 q_{1}+3 q_{2}-s\left(q_{0}-2 q_{1}+q_{2}\right)-q_{3}\right)- \\
& \frac{i\left(-(1-i s)^{n}+(1+i s)^{n}\right)\left(\left(-1+s^{2}\right) q_{0}-\left(-3+s^{2}\right) q_{1}-3 q_{2}+q_{3}\right)}{4 s^{3}\left(-1+s^{4}\right)}+ \\
& \frac{1}{4 s^{3}\left(-1+s^{4}\right)}(1-s)^{n}\left(s^{7}-q_{0}-s^{3}\left(1+q_{0}\right)+3 q_{1}+s^{2}\left(-q_{0}+q_{1}\right)-3 q_{2}-s\left(q_{0}-2 q_{1}+q_{2}\right)+q_{3}\right)-(14) \\
& \frac{1}{2} i\left(-(1-i s)^{n}+(1+i s)^{n}\right) \sum_{i=0}^{n}\left(-\frac{1}{4 s^{3}}\left((1-i s)^{i}+(1+i s)^{i}-i\left((1-i s)^{i}-(1+i s)^{i}\right) s\right)\left(1-s^{2}\right)^{1+i}\left(1-s^{4}\right)^{-1-i} q_{i}\right)+ \\
& \frac{1}{2}\left((1-i s)^{n}+(1+i s)^{n}\right) \sum_{i=0}^{n}\left(\frac{1}{4 s^{3}}\left(i\left((1-i s)^{i}-(1+i s)^{i}\right)+\left((1-i s)^{i}+(1+i s)^{i}\right) s\right)\left(1-s^{2}\right)^{1+i}\left(1-s^{4}\right)^{-1-i} q_{i}\right)+ \\
& (1+s)^{n} \sum_{i=0}^{n}\left(-\frac{(1-s)^{i}\left(1+s^{2}\right)^{i}\left(-1+s-s^{2}+s^{3}\right)\left(1-s^{4}\right)^{-1-i} q_{i}}{4 s^{3}}\right)+ \\
& (1-s)^{n} \sum_{i=0}^{n}\left(-\frac{(1+s)^{i}\left(1+s^{2}\right)^{i}\left(1+s+s^{2}+s^{3}\right)\left(1-s^{4}\right)^{-1-i} q_{i}}{4 s^{3}}\right) .
\end{aligned}
$$


Fig6: Eigenfunctions for the problem (11)-(12), $q(n)=n$

\begin{tabular}{|c|c|c|}
$n$ & $x(n), q(n)=\frac{1}{n+1}$ & $x(n), q(n)=\frac{1}{\sqrt{n+1}}$ \\
0 & 1 & 1 \\
1 & 1 & 1 \\
2 & 1 & 1 \\
3 & 1 & 1 \\
4 & 16.9467 & 2.0407 \\
5 & 80.7556 & 5.9615 \\
6 & 240.321 & 15.2739 \\
7 & 559.519 & 33.0829 \\
8 & 1373.36 & 63.0968 \\
9 & 4308.94 & 109.83 \\
10 & 14838.5 & 179.1 \\
11 & 47386.4 & 279.4 \\
12 & 138351 & 932.08 \\
13 & 393074 & 1377.6
\end{tabular}

\begin{tabular}{|c|c|c|}
$n$ & $x(n), q(n)=\frac{1}{n+1}$ & $x(n), q(n)=\frac{1}{\sqrt{n+1}}$ \\
0 & 1 & 1 \\
1 & 1 & 1 \\
2 & 1 & 1 \\
3 & 1 & 1 \\
4 & 5.8606 & 2.1832 \\
5 & 14.6518 & 6.6554 \\
6 & 31.0511 & 17.3085 \\
7 & 58.1807 & 37.7361 \\
8 & 100.113 & 72.3270 \\
9 & 162.983 & 126.80 \\
10 & 257.093 & 209.55 \\
11 & 400.414 & 820.25 \\
12 & 624.004 & 1289.4 \\
13 & 980.151 & 2043.5
\end{tabular}
Table4: Eigenfunctions correspond to the eigenvalue $\lambda=0.1296$

Fig7: Comparison of datas in Table3

Fig8: Comparison of datas in Table4

Proof. Firstly, we study to find the general solution of the equation (12) by the variation of parameters method without using the boundary conditions. Homogenous part of (12) is as follows,

$$
\Delta^{4} x(n)-\lambda x(n)=0 .
$$

By using delta exponential function, we have the characteristic equation,

$$
m^{4}-\lambda=0,
$$

and characteristic roots are as follows,

$$
m_{1,2}= \pm \sqrt[4]{\lambda}, m_{3,4}= \pm i \sqrt[4]{\lambda}
$$


where $m_{1,2,3,4} \in \Re$. So, the homogeneous solution is found from (44) as follows,

$$
x_{h}(n)=c_{1} e_{m_{1}}(n, 0)+c_{2} e_{m_{2}}(n, 0)+c_{3} e_{m_{3}}(n, 0)+c_{4} e_{m_{4}}(n, 0) .
$$

Suppose that

$$
\lambda=s^{4},
$$

and so, homogeneous solution is as follows by (5)

$$
x(n)=c_{1} e_{s}(n, 0)+c_{2} e_{-s}(n, 0)+c_{3} e_{\alpha}(n, 0) \cos _{\gamma}(n, 0)+c_{4} e_{\alpha}(n, 0) \sin _{\gamma}(n, 0),
$$

where $\alpha=0, \beta=s, \gamma=s$. If we insert $\alpha, \beta, \gamma$ in the equality above and using Theorem 2 and Definition 4 , then we have the homogenous solution of (12)

$$
x(n)=c_{1}(1-s)^{n}+c_{2}(1+s)^{n}+c_{3} \frac{(1+i s)^{n}+(1-i s)^{n}}{2}+c_{4} \frac{(1+i s)^{n}-(1-i s)^{n}}{2 i} .
$$

Then, if we apply the variation of parameters method and if we take the constants as parameters $v_{1}(n)$, $v_{2}(n), v_{3}(n), v_{4}(n)$, then we find the variables by Cramer rule,

$$
\begin{aligned}
& v_{1}=\sum_{i=0}^{n}\left(\frac{-2 s^{3}(1+s)^{i}\left(1+s^{2}\right)^{i}\left(1+s+s^{2}+s^{3}\right) q_{i}}{8 s^{6}\left(1-s^{4}\right)^{1+i}}\right) \\
& v_{2}=\sum_{i=0}^{n}\left(\frac{-2(1-s)^{i} s^{3}\left(1+s^{2}\right)^{i}\left(-1+s-s^{2}+s^{3}\right) q_{i}}{8 s^{6}\left(1-s^{4}\right)^{1+i}}\right) \\
& v_{3}=\sum_{i=0}^{n}\left(\frac{2 s^{3}\left(i\left((1-i s)^{i}-(1+i s)^{i}\right)+\left((1-i s)^{i}+(1+i s)^{i}\right) s\right)\left(1-s^{2}\right)^{1+i} q_{i}}{8 s^{6}\left(1-s^{4}\right)^{1+i}}\right) \\
& v_{4}=\sum_{i=0}^{n}\left(\frac{-2 s^{3}\left((1-i s)^{i}+(1+i s)^{i}-i\left((1-i s)^{i}-(1+i s)^{i}\right) s\right)\left(1-s^{2}\right)^{1+i} q_{i}}{8 s^{6}\left(1-s^{4}\right)^{1+i}}\right) .
\end{aligned}
$$

Applying the initial conditions (13), we have the constants as follows

$$
\begin{aligned}
& c_{1}=\frac{1}{4 s^{3}\left(-1+s^{4}\right)}\left(s^{7}-q_{0}-s^{3}\left(1+q_{0}\right)+3 q_{1}+s^{2}\left(-q_{0}+q_{1}\right)-3 q_{2}-s\left(q_{0}-2 q_{1}+q_{2}\right)+q_{3}\right), \\
& c_{2}=\frac{1}{4 s^{3}\left(-1+s^{4}\right)}\left(s^{7}+q_{0}-s^{3}\left(1+q_{0}\right)+s^{2}\left(q_{0}-q_{1}\right)-3 q_{1}+3 q_{2}-s\left(q_{0}-2 q_{1}+q_{2}\right)-q_{3}\right), \\
& c_{3}=\frac{s^{6}+q_{0}-s^{2}\left(1+q_{0}\right)-2 q_{1}+q_{2}}{2 s^{2}\left(-1+s^{4}\right)} \\
& c_{4}=\frac{\left(-1+s^{2}\right) q_{0}-\left(-3+s^{2}\right) q_{1}-3 q_{2}+q_{3}}{2 s^{3}\left(-1+s^{4}\right)} .
\end{aligned}
$$

Hence, we obtain the general solution (1) .

\section{Conclusion}

Consequently, the variation of parameters method for higher order linear ordinary difference equations with constant coefficient is considered with a new approachment by using delta exponential function. Analysis of the method is given in detailed and the advantage of this new approachment is to enable us to investigate the solution of difference equations in the closed form given in (2), otherwise it has to be expanded by using binomial expansion of the difference operator

$$
\Delta^{N} x(t)=\sum_{k=0}^{N}(-1)^{k}\left(\begin{array}{c}
N \\
k
\end{array}\right) x(t+N-k)
$$


and the method is supported with two difference eigenvalue problems; the second-order Sturm-Liouville (7) and the fourth-order relaxation difference equations (12). We find the sum representation of solution of Sturm-Liouville difference problem and also, we find the analytical solution of the fourth-order relaxation difference problem.

Moreover, behaviors of eigenfunctions for the problems (7) - (8) and (9) - (8) are analyzed and illustrated by graphics and tables.. Firstly, we show the behaviors of eigenfunctions while $q(n)=\frac{1}{\sqrt{n}}$ and eigenvalues are continuous in Fig1 and Fig2, and we observe that eigenfunctions are continous according to the eigenvalues. Also we compare the behaviors of eigenfunctions in Fig3. Then, we analyze the behaviors of eigenfunctions for the specific eigenvalues $\lambda=1$ and $\lambda=2-\sqrt{2}$ in Table1, Table 2 and Fig4 and Fig5 and we observe that eigenfunctions are discrete. We analyze the similar properties for the problem (12) - (13) in Fig6 while $q(n)=n$, in Fig7 and Table 3 while $\lambda=0.0625$, in Fig 8 and Table 4 while $\lambda=0.1296$.

\section{References}

[1] Adivar, M., Bairamov, E.: Spectral Properties of Non-selfadjoint Difference Operators, Journal of Mathematical Analysis and Applications, 261, 461-478 (2001).doi:10.1006/jmaa.2001.7532.

[2] Adivar, M., Bairamov, E.: Difference equations of second order with spectral singularities, J. Math. Anal. Appl. 277, 714-721 (2003).doi:10.1016/S0022-247X(02)00655-8.

[3] Bairamov, E., Aygar, Y., Koprubasi, T.: The spectrum of eigenparameter-dependent discrete Sturm-Liouville equations, Journal of Computational and Applied Mathematics, 235,4519-4523 (2011).doi:10.1016/j.cam.2009.12.037.

[4] K. Aydemir, O. S. Mukhtarov, Asymptotics eigenvalues for many-interval Sturm-Liouville problems, AIP Conference Proceedings, AIP Publishing, (2016), 1726:020088.

[5] Bas, Erdal, and Ramazan Ozarslan. "Sturm-Liouville Problem via Coulomb Type in Difference Equations." Filomat 31.4 (2017).

[6] Bas, Erdal, and Ramazan Ozarslan. "Asymptotics of eigenfunctions for Sturm-Liouville problem in difference equations." AIP Conference Proceedings. Eds. Theodore Simos, and Charalambos Tsitouras. Vol. 1738. No. 1. AIP Publishing, 2016.

[7] Bas, Erdal, and Ramazan Ozarslan. "Sturm-Liouville Difference Equations Having Special Potentials" Journal of Advanced Physics, Vol6, No4, 529-533 (2017).DOI10.1166/jap.2017.1360

[8] Bas, E., Metin, F.: Fractional singular Sturm-Liouville operator for Coulomb potential, Advances in Difference Equations, 300, (2013).DOI: 10.1186/1687-1847-2013-300.

[9] Ozturk, O., A study on nabla discrete fractional operator in mass -spring - damper system, New Trends in Mathematical Sciences, No. 4, 137-144 (2016).

[10] Ozturk, O. (2017). A study of $\nabla$-discrete fractional calculus operator on the radial Schrödinger equation for some physical potentials. Quaestiones Mathematicae, 1-11.

[11] C. Goodrich, A. C. Peterson. Discrete fractional calculus, Berlin, Springer, (2015).

[12] Kelley, W.G., Peterson, A.C.: Difference Equations: An Introduction with Applications, Academic Press, San Diego (2001).

[13] Bender, C.M., Orszag, S.A.: Advanced Mathematical Methods for Scientists and Engineers: Asymptotic Methods and Perturbation Theory, Springer-Verlag, Newyork (1999). 
[14] Gulsen, T., \& Yilmaz, E. (2017). Spectral theory of Dirac system on time scales. Applicable Analysis, 96(16), 2684-2694.

[15] Lagrange, J. L. (1810). Second mémoire sur la théorie générale de la variation des constantes arbitraires dans tous les problemes de la mécanique. Lu, le, 19, 809-816.

[16] Euler, L. (1753). Theoria motus lunae eschibens omnes eius inaequalitates in additamento hoc idem argumentum aliber tractatur simulaque... Acadeniae Inperialis Scientiarum Petropolitanae.

[17] W. N. Everitt, A catalogue of Sturm-Liouville differential equations. In Sturm-Liouville Theory. Birkhäuser Basel;2005, pp. 271-331

[18] C. J. Adkins, Sturm-Liouville Theory, MSc thesis, University of Toronto; 2014.

[19] E. S. Panakhov, I. Ulusoy, Asymptotic behavior of eigenvalues of hydrogen atom equation. Boundary Value Problems, 2015;2015(1): 1-15.

[20] E. Bas, E. Panakhov, R. Yllmazer, The Uniqueness Theorem for Hydrogen Atom Equation. TWMS Journal of Pure and Applied Mathematics, 2013;4:20-28.

[21] D. I. Blohincev, Foundations of Quantum Mechanics. GITTL, Moscow, 1949. 